\title{
La repercusión del bilingüismo en el rendimiento académico en alumnos de colegios públicos de la Comunidad de Madrid $^{1}$
}

\author{
The impact of bilingual education in academic \\ achievement of students enrolled in public schools in \\ the Autonomous Community of Madrid \\ Esmeralda SOTOCA SIENES \\ CEIP Ángel Berzal Fernández, Madrid
}

Recibido: Abril 2013

Aceptado: Julio 2013

\begin{abstract}
Resumen
El objetivo del presente estudio es analizar si existen diferencias en el rendimiento académico de alumnos de segundo y tercer ciclo de Educación Primaria en función de su asistencia a colegios públicos bilingües o no de la Comunidad de Madrid. Para lograr el objetivo se ha trabajado con un diseño cuasiexperimental con grupo de control no-equivalente. La muestra estuvo formada por 2.153 alumnos de $3^{\circ}$ a $6^{\circ}$ de Educación Primaria de 12 colegios públicos de la Comunidad de Madrid pertenecientes a la Dirección de Área Territorial Madrid-Este. El rendimiento académico se ha medido mediante calificaciones escolares y pruebas externas (Evaluación de Diagnóstico de $4^{\circ}$ de E.P. y Prueba CDI de $6^{\circ}$ de E.P). En relación con la prueba CDI, se han hallado diferencias significativas a favor de los colegios bilingües en Lengua y en Matemáticas. En la prueba externa de $4^{\circ}$ de E.P. no hay diferencias significativas. Respecto a las calificaciones escolares, se han hallado diferencias significativas a favor de los colegios no bilingües en Conocimiento del Medio e Inglés.
\end{abstract}

Palabras clave: bilingüismo; rendimiento académico; aprendizaje de segunda lengua; Educación Primaria (E.P.).

\begin{abstract}
The purpose of this study is to analyze if there are differences in academic achievement among students in the second and third cycles of Primary Education in bilingual or non-bilingual public schools in Madrid. A quasi-experimental approach has been adopted with nonequivalent control group. The sample consisted of 2.153 students, Years 3 to 6 Primary Education enrolled in 12 public schools located in the eastern part of Madrid. The academic achievement is measured by Grade Point Average (GPA) and external examinations (Diagnosis Test for Year 4 and CDI
\end{abstract}

\footnotetext{
${ }^{1}$ Parte de esta investigación se llevó a cabo durante el disfrute de una licencia por estudios concedida durante el curso 2009/10. 
Test for Year 6). From the results, the external tests show significant differences in favour of bilingual schools in Language and Mathematics in Year 6. But there were no significant differences in Year 4. However, in the GPA, there are significant differences in favour of nonbilingual schools in Science and English.

Keywords: bilingual education; academic achievement; second language learning; Primary Education.

Durante los últimos años ha surgido en España un número considerable de proyectos bilingües en Educación Primaria con el objetivo de mejorar el aprendizaje de lenguas extranjeras.

La educación bilingüe cuenta con una larga tradición en zonas bilingües de EE.UU., Canadá, Países Bajos, etc. Sin embargo, en España es una práctica mucho más novedosa (en el presente estudio no se hace referencia a los programas de inmersión lingüística de las Comunidades Autónomas que poseen una lengua autonómica), debido a que su implantación en comunidades de habla castellana con una sola lengua oficial es muy reciente.

En la Comunidad de Madrid (en adelante, C.M.) actualmente existen dos proyectos bilingües distintos en los colegios públicos. Por una parte está el Programa de Colegios Bilingües, objeto del presente estudio $\mathrm{y}$, por otra, 10 colegios públicos en la Comunidad de Madrid imparten enseñanza bilingüe español-inglés a través del Convenio suscrito en 1996 entre el Ministerio de Educación y Ciencia y el British Council (Currículo integrado hispano británico).

En referencia al primer proyecto, en marzo de 2004 aparece la primera orden donde se daba a conocer la implantación de los colegios públicos bilingües español-inglés en la C.M. La orden 796/2004 expone que se entiende por enseñanza bilingüe la que permite impartir, como mínimo, un tercio del horario lectivo semanal en lengua inglesa. De esta forma, los centros bilingües incrementan hasta cinco horas semanales el tiempo dedicado al Área de Lengua Extranjera y se posibilita la impartición en inglés de otras Áreas curriculares, con la excepción de las Áreas de Matemáticas y Lengua Castellana.

Educación Física, Educación Artística y Conocimiento del Medio son áreas que enriquecen el Proyecto al poder impartirse en inglés, dotando a los colegios de un estímulo lingüístico adicional, hasta completar el mínimo del 30\% del tiempo establecido en la docencia directa.

La concepción pedagógica del Programa se corresponde con el modelo AICOLE, es decir, Aprendizaje Integrado de Contenidos y Lengua, que consiste en la utilización de una segunda lengua en el proceso de aprendizaje de conocimientos y contenidos. En concreto, los alumnos del Programa Bilingüe utilizan el español y el inglés como lenguas vehiculares de aprendizaje, se trata de aprender los contenidos con independencia de la lengua que se utilice.

El Programa de Colegios Bilingües de la Comunidad de Madrid se desarrolla en los colegios públicos de Educación Infantil y Primaria seleccionados por la Consejería de 
Educación para su implantación, comenzando en el primer curso de Educación Primaria e implantándose progresivamente (un curso más cada año) en el resto de los cursos hasta comprender toda la Educación Primaria. Dicho programa abarca centros completos y no partes o líneas de los mismos, como ocurre en el modelo de Secciones que es el más extendido en otras Comunidades Autónomas.

Comenzaron siendo 26 los centros públicos seleccionados por la Consejería de Educación y, actualmente, dicha cifra se eleva a 298 (en el curso 2012-2013).

Sin embargo, se considera indispensable la evaluación del Programa Bilingüe de la C.M. en Primaria, debido a que desde su implantación únicamente se ha realizado una evaluación en referencia a la Prueba de Conocimientos y Destrezas Indispensables (en adelante, CDI) en $6^{\circ}$ de Educación Primaria por parte del Consejo Escolar de la Comunidad de Madrid (2010).

Se trata de un proyecto que se encuentra en las primeras fases de su desarrollo y que requiere una evaluación de los resultados obtenidos. La Federación de la C.M. de Asociaciones de Padres y Madres Francisco Giner de los Ríos (2009) señala que el programa de bilingüismo requiere una evaluación rigurosa de los resultados académicos, comparándolos con los obtenidos por los centros no bilingües en las diferentes materias impartidas, ya lo sean en Inglés o en Castellano.

De esta forma, con la presente investigación se pretende profundizar un poco más en referencia a los efectos de la implantación de dicho programa de la C.M. en el rendimiento académico de los alumnos. En el presente trabajo el rendimiento se ha medido a través de las calificaciones escolares (recogidas de los informes y las actas de evaluación final), los resultados de la prueba de evaluación externa "Conocimientos y Destrezas Indispensables" que realiza la C.M. en todos los colegios en $6^{\circ}$ de Educación Primaria (en adelante, E.P.), y la prueba de diagnóstico de $4^{\circ}$ de E.P. que también se lleva a cabo en dicha comunidad. La muestra es de 2.153 alumnos de 12 colegios de la Dirección del Área Territorial de Madrid-Este, con edades comprendidas entre los 8 y 13 años.

El objetivo de la presente investigación es comprobar si existen diferencias entre centros públicos bilingües y no bilingües ${ }^{2}$ de la Comunidad de Madrid en el rendimiento académico (áreas de Lengua, Matemáticas, Conocimiento del Medio e Inglés) en alumnos de segundo y tercer ciclo de E.P.

\footnotetext{
${ }^{2}$ Los alumnos de los colegios no bilingües han seguido una educación monolingüe a lo largo de la etapa de E.P. y han estudiado la L2/LE (Lengua Extranjera) como una asignatura más del currículo escolar. La lengua materna (L1) o primera lengua es aquella que se aprende desde la infancia. La segunda lengua (L2) es la lengua que se aprende después de que se ha aprendido la primera lengua y, por lo tanto, se cuenta ya con un sistema lingüístico en el cerebro.
} 


\section{Fundamentación teórica}

En relación con la definición de educación bilingüe, la normativa vigente sobre centros bilingües de la C.M. únicamente señala en la orden 796/2004 que se entiende por enseñanza bilingüe la que permite impartir, como mínimo, un tercio del horario lectivo semanal en lengua inglesa.

Cummins (2010) sostiene que la educación bilingüe se refiere al uso de dos lenguas de instrucción en algún punto de la carrera escolar de un estudiante, utilizando cada lengua como medio de instrucción para enseñar el contenido de la asignatura más que sólo el lenguaje en sí mismo. No incluye programas escolares donde se enseña una segunda lengua solamente como una asignatura. Cummins (2002) señala que el objetivo no es que el alumno que adquiere la lengua logre el nivel de un hablante nativo. Expone que se debe buscar un dominio de la segunda lengua que permita al alumno utilizarla de manera adecuada en el contexto escolar: "El constructo del dominio académico del idioma no alude a una idea absoluta del dominio de uso del idioma, sino al grado en el que una persona tiene acceso y maestría suficiente para comprender y utilizar el tipo concreto de lenguaje empleado en contextos educativos necesario para realizar las tareas académicas" (Cummins, 2002, p. 83-84).

A continuación se va a realizar un análisis de los estudios que respaldan la relación entre el bilingüismo y el incremento del rendimiento académico. En primer lugar se van a señalar estudios que respaldan la correlación entre el bilingüismo y el incremento del rendimiento académico, en segundo lugar investigaciones sobre bilingüismo y rendimiento en producción escrita, en tercer lugar se señalarán estudios que exponen que el bilingüismo es beneficioso para el desarrollo de habilidades de lectura de los estudiantes $\mathrm{y}$, en cuarto lugar, investigaciones que afirman que el bilingüismo es beneficioso para el desarrollo de habilidades matemáticas de los estudiantes.

En primer lugar, en relación con las investigaciones sobre la correlación entre el bilingüismo y el incremento del rendimiento académico (utilizando tanto pruebas estandarizadas como certificaciones académicas u otras medidas), se puede señalar que existen numerosas investigaciones que han demostrado los beneficios académicos que obtienen los alumnos bilingües en relación a los monolingües.

El Consejo Escolar de la Comunidad de Madrid (2010) realizó un estudio en relación con la evaluación externa del rendimiento de los alumnos del Programa Bilingüe de la Comunidad de Madrid, centrándose únicamente en el efecto indirecto de la variable centro sobre el rendimiento de los alumnos de sexto curso de Educación Primaria, medido a través de la prueba CDI. Los datos de dicho estudio mostraron que los centros bilingües puntuaron sistemáticamente por encima de los no bilingües en las pruebas CDI, siendo las diferencias entre uno y otro grupo estadísticamente significativas tanto en Lengua como en Matemáticas.

Johnstone, Dobson y Pérez Murillo (2010) realizaron una evaluación externa del currículo integrado en centros públicos españoles, fruto del convenio realizado entre el Ministerio de Educación y Ciencia y el British Council. Estos autores exponen que la mayoría del alumnado tiene un buen rendimiento en clase y alcanza un nivel excelente 
en su expresión oral y escrita en inglés; en su expresión escrita en español, en la que obtuvieron mejores resultados que los alumnos del mismo centro que no participaban en el proyecto bilingüe, y en sus resultados en las pruebas IGCSE (International General Certificate of Secondary Education). Las pruebas IGCSE de 2009 mostraron que los estudiantes mejoraron sus logros en áreas como Historia, Geografía o Biología, cuyos exámenes se realizan en inglés, y que alcanzaron un rendimiento muy alto en la prueba de Español 1 (concebida para hispanohablantes).

Otros estudios no centrados en los programas bilingües de la C.M. igualmente señalan la superioridad de los sujetos bilingües respecto a los monolingües en relación al rendimiento académico (Barik y Swain, 1978; Cohen, 1974; Cummins, 2001; Genesee et al., 1986; Kessler y Quinn, 1980; Swain y Lapkin, 1981; Thomas y Collier, 2003; Thomas, Collier y Abbott, 1993). Investigaciones con sujetos trilingües también muestran resultados similares (por ejemplo, Genesee y Lambert, 1983; y Lasagabaster, 2005).

También numerosos autores como Genesee (1987); Halsall (1998); Holobow, Genesee, Lambert y Gastright (1987); Lapkin, Swain y Shapson (1990); Turnbull, Lapkin y Hart (2001), sostienen que la competencia en la primera lengua de los alumnos de inmersión es equiparable a la de los alumnos que estudian en clases monolingües, y que los resultados en las otras asignaturas no se ven afectados por el hecho de ser impartidas en una segunda lengua, además de que estos alumnos adquieren una competencia mucho mayor en la segunda lengua.

Se deben citar además otros estudios que se han centrado en el número de años que necesitan los alumnos bilingües para igualar o superar a los monolingües en rendimiento. Turnbull, Hart y Lapkin (2003) hallaron que cualquier retraso en el logro de los alumnos de inmersión en lectura, escritura y Matemáticas desaparece en $6^{\circ}$ curso. Edwards (1989) señala que hay algunos retrasos en las destrezas en lengua inglesa en los alumnos de inmersión en los cursos de $1^{\circ}$ a $3^{\circ}$, y que dichos retrasos desaparecen hacia el $4^{\circ}$ ó $5^{\circ}$ curso. Wayne y Collier (2002) sostienen que los estudiantes escolarizados en programas bilingües superan a los estudiantes comparables escolarizados de forma monolingüe en rendimiento en todas las asignaturas, después de 4-7 años de educación en inmersión recíproca. Cade (1997) afirma que, con el paso del tiempo, los estudiantes de la segunda lengua mejoran las puntuaciones en las pruebas y tienen más éxito en su primera lengua.

Pagan (2005) expone que el bilingüismo beneficia tanto a los alumnos de la lengua mayoritaria como a los de la minoritaria.

En segundo lugar, en relación al bilingüismo y el rendimiento en producción escrita, aparte de las investigaciones expuestas anteriormente en las que los alumnos bilingües superaban a los monolingües en escritura (Johnstone, Dobson y Pérez Murillo, 2010; Turnbull, Hart y Lapkin, 2003), se recogen a continuación otros estudios que han hallado resultados semejantes. Bialystok (1986) y Bialystok y Majumder (1998) señalan que el bilingüismo incide positivamente en el desarrollo del lenguaje escrito. 
Galindo y Moreno (2008) concluyeron que la superioridad de los alumnos bilingües sobre los monolingües en la producción argumentativa escrita en lengua materna, dependió de su avance en la escolaridad. En este sentido, Bialystok (1986), Bialystok y Majumder (1998), Cromdal (1999) y Galindo y Moreno (2008) afirman que los alumnos bilingües de mayor curso escolar, con mayor experiencia escolar, son más competentes que sus iguales bilingües de menor curso escolar en la resolución de problemas relativos a la reflexión y la manipulación del componente sintáctico de la lengua, es decir, en la habilidad metasintáctica.

Swain (1987) y Swain y Lapkin (1982) señalan que durante el primer y segundo curso de E.P. los alumnos bilingües consiguen una producción de textos narrativos escritos en lengua materna inferior a la de sus homólogos escolarizados en centros educativos monolingües. Sin embargo, expone Lapkin (1982) que esta inferioridad es transitoria, debido a que la producción escrita en lengua materna de los alumnos bilingües en inmersión es igual a la de los monolingües a partir del momento en que se introduce la lengua materna en el currículo escolar, generalmente, en el segundo o tercer curso de E.P. En $6^{\circ}$ de E.P. los alumnos bilingües en inmersión logran una producción escrita en lengua materna de textos narrativos igual o superior a la de sus iguales monolingües (Lapkin, 1982; Lambert et al., 1993; Turnbull, Lapkin y Hart, 2001).

En tercer lugar se van a analizar las aportaciones entorno a si el bilingüismo es beneficioso para el desarrollo de las habilidades de lectura de los estudiantes. Además del estudio citado anteriormente de Turnbull, Hart y Lapkin (2003) que señalaba la superioridad en la lectura de los sujetos bilingües sobre los monolingües, otras investigaciones han obtenido resultados similares. Así, Bialystok (1997), D'Angiulli, Siegel y Serra (2001), Demont (2001) y Kimbrough Oller y Cobo-Lewis (2002) obtuvieron resultados superiores por parte de los alumnos bilingües en distintas habilidades lectoras. Ricciardelli (1993) halló que los alumnos bilingües que demostraron alta competencia en las dos lenguas alcanzaron unas puntuaciones más altas en pruebas de rendimiento en lectura.

Sin embargo, Hawson (1996) señala que los aprendices de segunda lengua en situaciones de inmersión experimentan un cambio atencional desde el procesamiento del sistema auditivo hacia el procesamiento de la información visual, favoreciendo esto el aprendizaje en materias que son fácilmente procesadas vía análisis visual, mientras que puede inhibir el aprendizaje en materias que dependen del procesamiento vía sistema auditivo. Debido a que la lectura depende de la conciencia fonológica, la habilidad de lectura de estos alumnos puede sufrir y consecuentemente, materias que dependen de la lectura para la adecuada competencia pueden estar negativamente afectadas.

En cuarto lugar se van a señalar algunas investigaciones que exponen que el bilingüismo es beneficioso para el desarrollo de las habilidades matemáticas de los estudiantes. Pifarré et al. (2003) señalan que ciertos modelos de educación bilingüe, al promover competencias en dos o más lenguas, no sólo promueven una mayor competencia lingüística, sino que pueden incidir también en el aprendizaje de 
habilidades y conocimientos matemáticos. Bournot-Trites y Reeder (2001) y Lambert et al. (1993) investigaron el efecto que tenía enseñar Matemáticas en la L2 en la evaluación del rendimiento de Matemáticas administrado en la L1, y hallaron que los grupos que mejores resultados obtuvieron recibieron una instrucción en la L2 más intensiva que los otros grupos.

Finalmente, Hawson (1996) justifica el alto rendimiento en Matemáticas por parte de estudiantes de inglés como segunda lengua señalando que Matemáticas es un área de contenido fácilmente analizada de forma visual, y los aprendices de segunda lengua desarrollan una intensificada capacidad para procesar información por medio del sistema visual debido a la sobrecarga del procesamiento del sistema auditivo y a la familiaridad de la entrada visual en el entorno.

\section{Metodología de la investigación}

La hipótesis del presente estudio es que existirán diferencias estadísticamente significativas en rendimiento escolar en todas las materias señaladas (Lengua, Matemáticas, Conocimiento del Medio e Inglés), con independencia de la prueba de rendimiento utilizada para medir, a favor de los centros bilingües.

Este estudio utilizó un diseño cuasiexperimental con grupo de control noequivalente, comparando los resultados de los alumnos de colegios públicos bilingües de la C.M. con un grupo similar de alumnos de colegios públicos no bilingues.

\section{Participantes}

La muestra estuvo formada por 2.153 alumnos de $3^{\circ}$ a $6^{\circ}$ de Educación Primaria de 12 colegios públicos de la Comunidad de Madrid pertenecientes a la Dirección de Área Territorial Madrid-Este, de los cuales un 49,2 \% fueron mujeres y un 50,8 \% varones. El nivel cultural y socioeconómico de los alumnos era variable, incluyéndose alumnado de la zona rural y urbana. Los centros participantes fueron todos los colegios bilingües de la primera y segunda convocatorias de centros bilingües de la Dirección de Area Territorial Madrid-Este que accedieron a participar en el estudio voluntariamente, se trata por tanto de una muestra incidental en la que seis centros bilingües decidieron participar y posteriormente se intentaron emparejar estos seis colegios con centros no bilingües de semejantes características (según el tipo de alumnado, nivel socio-cultural, zona rural-no rural, tamaño del centro, proximidad física en la ubicación de los centros, etc.). De modo que, finalmente, participaron 12 centros pertenecientes todos ellos a la DAT de Madrid-Este.

Se exponen, seguidamente, los gráficos de distribución de la muestra en función de las variables socio-demográficas, que muestran los datos señalados anteriormente. 


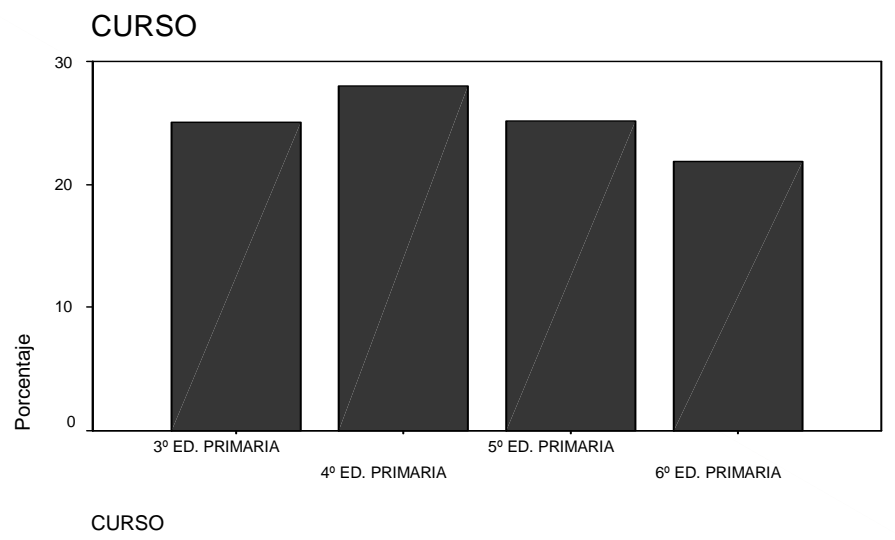

Gráfico 1. Distribución de la muestra en función del curso.

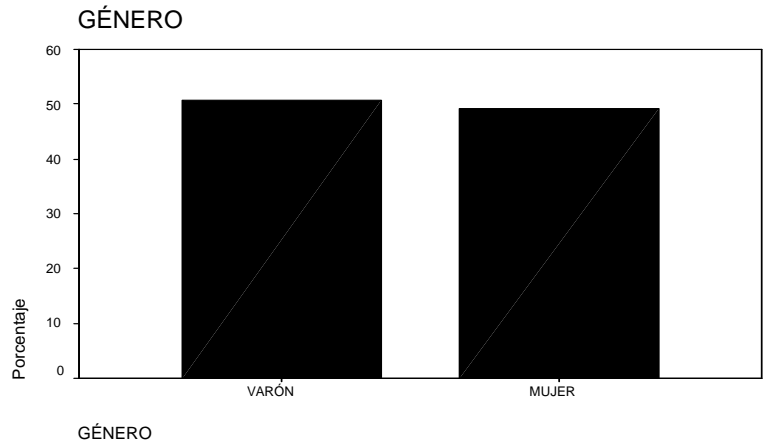

Gráfico 2. Distribución de la muestra en función del género.

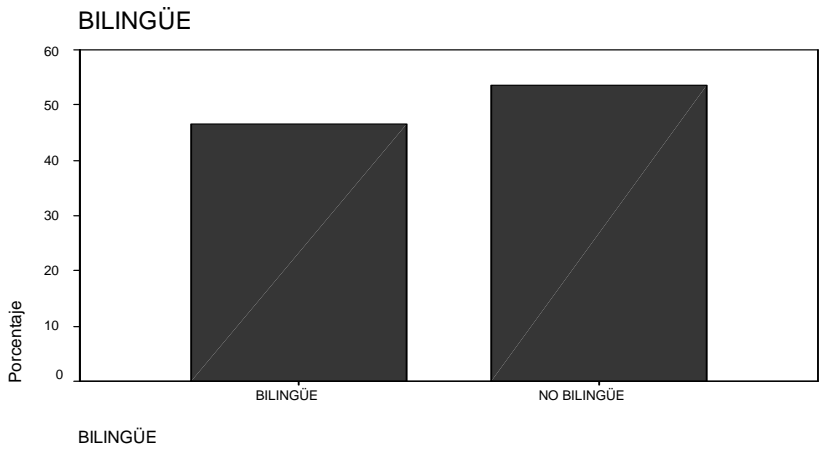

Gráfico 3. Distribución de la muestra en función de que el centro sea bilingüe o no bilingüe.

No obstante, a lo anteriormente expuesto se debe añadir que la muestra de los alumnos de $6^{\circ}$ de E.P. es más reducida debido a que no se recogieron los resultados de 
los alumnos que no habían cursado todavía enseñanzas bilingües durante ese curso. Es decir, en los centros en los que todavía no había sido implantado el bilingüismo en $6^{\circ}$ de E.P., no se recogieron los resultados académicos y las pruebas CDI, debido a que esos niños no estaban cursando las enseñanzas bilingües durante el curso escolar 2009/2010. Por ese motivo, en el Gráfico 1 se observa una disminución del alumnado de $6^{\circ}$ de E.P.

\section{Instrumentos}

Para la medición del rendimiento académico se utilizaron:

- Las actas e informes de evaluación final de los alumnos de $3^{\circ}$ a $6^{\circ}$ de E.P. (calificaciones escolares).

- Los resultados de la prueba de diagnóstico de $4^{\circ}$ de E.P. y de la prueba C.D.I. de $6^{\circ}$ de E.P. de la C.M. (pruebas externas).

\section{Calificaciones escolares (recogidas de las actas e informes de evaluación final)}

Las calificaciones son el criterio social y legal del rendimiento académico de un alumno en el ámbito institucional. Normalmente se establecen mediante exámenes que pueden presentar limitaciones tanto en su elaboración como en los criterios de corrección, pues es inevitable la subjetividad del profesor.

En esta investigación se consideran seis niveles de desempeño académico, correspondientes a los criterios empleados en los centros según la normativa vigente sobre evaluación en la Comunidad de Madrid: Insuficiente, Suficiente, Bien, Notable, Sobresaliente y Mención Honorífica. Los niveles de desempeño académico se obtuvieron a través de las calificaciones escolares (actas e informes de evaluación final). En dichos documentos aparece el resultado de una evaluación cualitativa que tiene en cuenta seis categorías, pero para realizar los pertinentes análisis estadísticos, éstas se transformaron en números del 1 al 6 , siendo Insuficiente el equivalente a 1 y Mención Honorífica el equivalente a 6 . De esta forma, se ha utilizado una escala del 1 al 6 para expresar las calificaciones escolares (1=Insuficiente, $\quad 2$ =Suficiente, $\quad 3=$ Bien, $\quad 4=$ Notable,$\quad 5=$ Sobresaliente, $\quad 6=$ Sobresaliente con Mención Honorífica).

\section{Prueba de Conocimientos y Destrezas Indispensables (CDI)}

La Prueba de Conocimientos y Destrezas Indispensables es una prueba censal de carácter externo que realiza la Consejería de Educación de la C.M. al finalizar $6^{\circ}$ de Educación Primaria que va dirigida a todos los alumnos de sexto curso de Educación Primaria de todos los centros de la Comunidad de Madrid.

Debido a que en el presente estudio se recogieron los resultados de dicha prueba del curso 2009/2010, la normativa por la que se rigió la aplicación de la prueba fue la RESOLUCIÓN de 15 de marzo de 2010. Dicha Resolución establecía que la prueba y los correspondientes criterios de corrección eran elaborados por la Dirección General de Educación Infantil y Primaria y que la prueba versaba sobre Lengua Castellana y Matemáticas. Se estructuraba en dos partes. La primera constaba de un dictado, la lectura de un texto y las respuestas a cinco preguntas 
relativas al mismo y cinco preguntas de cultura general. La segunda parte constaba de diez ejercicios y cinco problemas sencillos de Matemáticas.

La corrección de la prueba fue realizada en las Direcciones de Área Territorial por comisiones previamente nombradas por los respectivos Directores de Área.

En referencia a los resultados de la prueba CDI de $6^{\circ}$ de E.P., los datos se expresaron en una escala del 0 al 20.

\section{Evaluación de Diagnóstico de $4^{\mathrm{o}}$ de Educación Primaria}

Al finalizar el $4^{\circ}$ curso de Primaria se realizan evaluaciones de diagnóstico con carácter interno para todos los alumnos y externo para una muestra convenientemente seleccionada, que consisten en pruebas elaboradas por la Consejería de Educación dirigidas a todos los alumnos de la Comunidad de Madrid sobre contenidos "curriculares" en Lengua y Matemáticas.

La RESOLUCIÓN de 26 de marzo de 2010 señalaba que dicha evaluación contenía una prueba de Matemáticas y otra de Comunicación Lingüística, que constó a su vez de dos partes: una de comprensión lectora y otra sobre competencias curriculares de la materia de Lengua Castellana y Literatura que incluía un dictado.

La Dirección General de Mejora de la Calidad de la Enseñanza fue la responsable de la elaboración y difusión de las pruebas.

En relación con la prueba de diagnóstico de $4^{\circ}$ de E.P., los datos se expresaron en porcentajes $(0-100)$.

\section{Resultados}

Los resultados presentados se basan en contraste de hipótesis basado en pruebas paramétricas y muestras independientes, comparando los grupos bilingües y no bilingües y considerando los tres tipos de medidas del rendimiento.

\section{Consideraciones previas en referencia a los instrumentos utilizados}

Previamente a la realización del contraste de hipótesis se va a comprobar que las pruebas miden el mismo constructo. De este modo, las correlaciones que aparecen a continuación se han realizado para determinar que tanto las pruebas externas (prueba de diagnóstico de $4^{\circ}$ de E.P. y prueba CDI de $6^{\circ}$ de E.P.) como las notas (recogidas de las actas e informes de evaluación final) miden lo que tienen que medir, y como consecuencia de que eso es verídico, se han empleado ambos tipos de datos en el presente estudio.

En primer lugar, en referencia a la correlación entre la prueba de diagnóstico de $4^{\circ}$ E.P. y el rendimiento académico medido a través de las actas de evaluación final, en la Tabla I se puede observar que las correlaciones son medias y significativas $(\mathrm{p}<, 01)$. 


\begin{tabular}{|c|c|c|c|c|}
\hline & $\begin{array}{c}\text { Prueba } 4^{\circ} \\
\text { Matemáticas } \\
\end{array}$ & $\begin{array}{c}\text { Prueba } 4^{\circ} \\
\text { Lengua } \\
\end{array}$ & Lengua & Matemáticas \\
\hline $\begin{array}{l}\text { Prueba } 4^{\circ} \\
\text { Matemáticas }\end{array}$ & 1 & & & \\
\hline Prueba $4^{\circ}$ Lengua & $\begin{array}{c}, 667 \\
(, 000) \\
\end{array}$ & 1 & & \\
\hline Lengua & $\begin{array}{l}, 575 \\
(, 000) \\
\end{array}$ & $\begin{array}{l}, 621 \\
(, 000)\end{array}$ & 1 & \\
\hline Matemáticas & $\begin{array}{l}, 701 \\
(, 000)\end{array}$ & $\begin{array}{l}, 607 \\
(, 000)\end{array}$ & $\begin{array}{l}, 810 \\
(, 000)\end{array}$ & 1 \\
\hline
\end{tabular}

Tabla I. Correlación entre la prueba de Diagnóstico de $4^{\circ}$ de E.P. y las calificaciones escolares.

En segundo lugar, en referencia a la correlación entre la prueba CDI de $6^{\circ}$ E.P. y el rendimiento académico medido a través de las actas de evaluación final, en la Tabla II se observa que las correlaciones son medias y significativas.

\begin{tabular}{lcccc}
\hline & $\begin{array}{c}\text { CDI } \\
\text { Matemáticas }\end{array}$ & CDI Lengua & Lengua & Matemáticas \\
\hline CDI Matemáticas & 1 & & & \\
\hline CDI Lengua &, 630 & 1 & 1 & \\
\hline Lengua & $(, 000)$ & & & \\
\hline Matemáticas &, 599 &, 714 & $(, 000)$ & \\
\hline
\end{tabular}

Tabla II. Correlación entre la prueba CDI de $6^{\circ}$ de E.P. y las calificaciones escolares.

\section{Resultados basados en las calificaciones escolares}

La tabla que aparece a continuación muestra el porcentaje de sujetos que se incluye en cada categoría de rendimiento en las áreas de Conocimiento del Medio, Lengua, Inglés y Matemáticas basado en las calificaciones escolares (Insuficiente, Suficiente, Bien, Notable, Sobresaliente y Sobresaliente con Mención Honorífica). Como se puede observar en ella, el porcentaje más alto se sitúa en el notable en todas las áreas consideradas y las calificaciones con porcentajes más bajos se sitúan en el sobresaliente con mención honorífica. 


\begin{tabular}{lcccc}
\hline & $\begin{array}{c}\text { Conocimiento } \\
\text { del medio }\end{array}$ & Lengua & Inglés & Matemáticas \\
\hline Insuficiente & 17,6 & 13,0 & 15,5 & 15,6 \\
Sufiente & 19,4 & 17,5 & 19,2 & 19,0 \\
Bien & 16,9 & 20,9 & 17,9 & 20,2 \\
Notable & 27,4 & 29,1 & 26,1 & 29,2 \\
Sobresaliente & 17,5 & 17,8 & 20,2 & 14,1 \\
Mención honorífica & 1,2 & 1,7 & 1,1 & 2,0 \\
\hline
\end{tabular}

Tabla III. Porcentaje de sujetos que se incluye en cada categoría de rendimiento en las diferentes áreas basado en las calificaciones escolares.

Resultados basados en las calificaciones escolares

\begin{tabular}{lcccc}
\hline & $\begin{array}{c}\text { Conocimiento } \\
\text { del medio * }\end{array}$ & Lengua & Inglés* & Matemáticas \\
\hline Bilingüe & 3,1 & 3,3 & 3,2 & 3,2 \\
No bilingüe & 3,2 & 3,3 & 3,3 & 3,1 \\
\hline
\end{tabular}

Nota: El * indica que la diferencia de medias es significativa al nivel de confianza del $95 \%$.

Tabla IV. Estadísticos de grupo en función de la variable bilingüe-no bilingüe en rendimiento (medido con las calificaciones escolares).

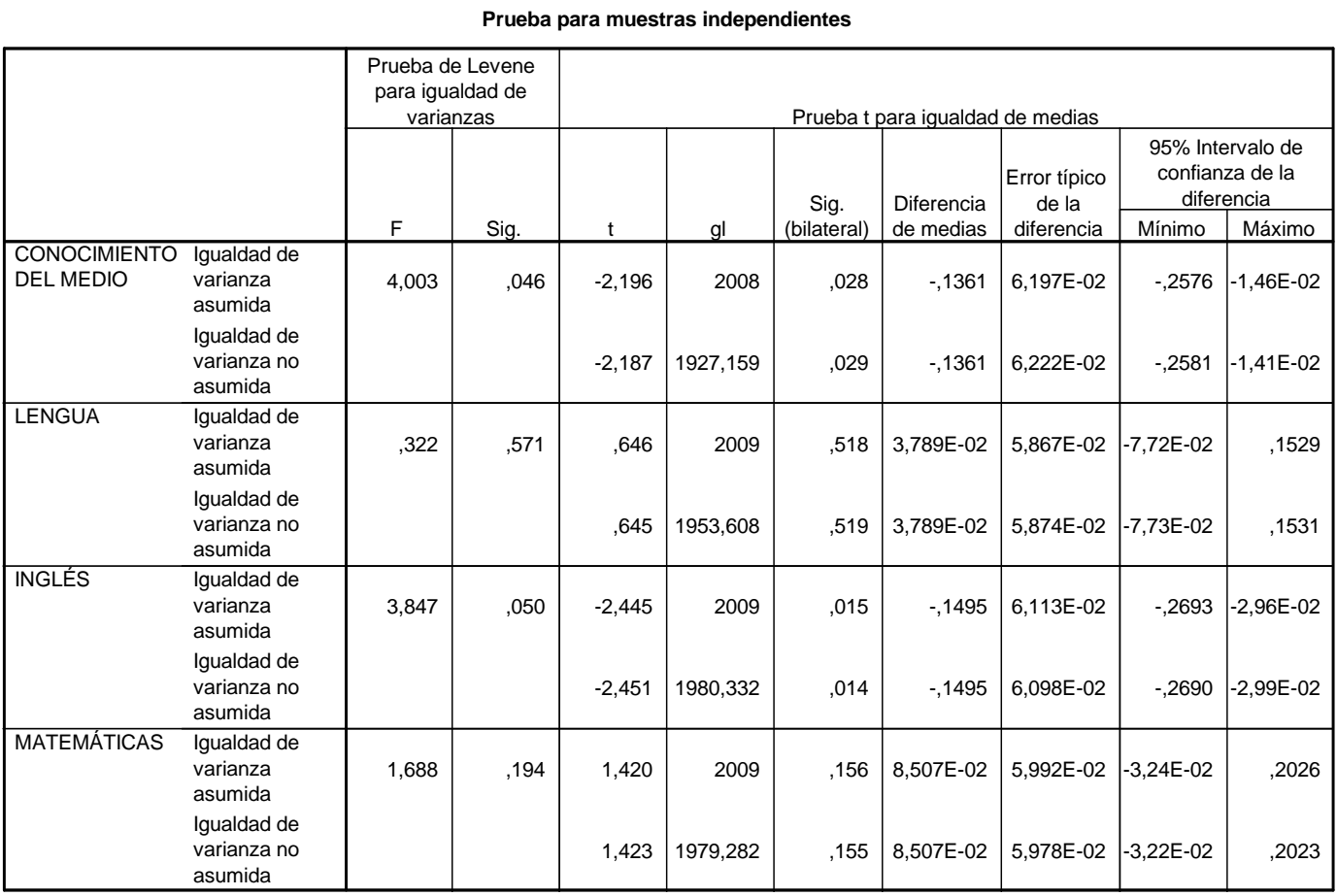

Tabla V. Prueba para muestras independientes en función de la asistencia a centro bilingüe-no bilingüe en rendimiento (medido con las calificaciones escolares). 
En los resultados de la Tabla $\mathrm{V}$ se hallan diferencias estadísticamente significativas $(p<, 05)$ en las variables Inglés $(, 015)$ y Conocimiento del Medio $(, 028)$, observando que los colegios no bilingües obtienen unas puntuaciones más altas (en la Tabla IV los centros no bilingües tienen en Inglés una media de 3,3 y en Conocimiento del Medio de 3,2$)$.

Resultados basados en las pruebas de diagnóstico de $4^{\circ}$ E.P.

\begin{tabular}{lcccc}
\hline & \multicolumn{2}{c}{$\begin{array}{c}\text { Prueba 4 } \\
\text { Matemáticas }\end{array}$} & \multicolumn{2}{c}{$\begin{array}{c}\text { Prueba 4 } \\
\text { Lengua }\end{array}$} \\
\hline Bilingüe & $\mathrm{N}$ & Media & $\mathrm{N}$ & Media \\
No bilingüe & 259 & 64,5 & 257 & 72,3 \\
\hline
\end{tabular}

Tabla VI. Estadísticos de grupo en función de la variable bilingüe-no bilingüe en rendimiento (medido con la prueba de diagnóstico de $4^{\circ}$ de E.P.).

Prueba para muestras independientes

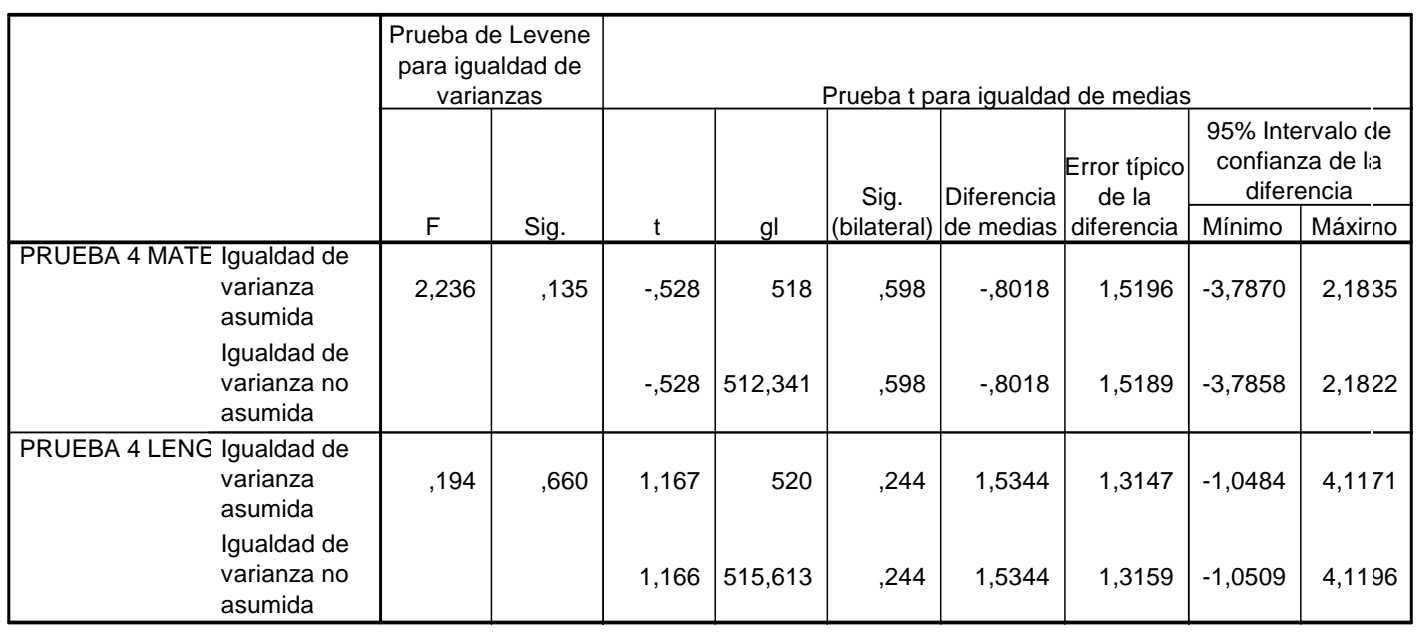

Tabla VII. Prueba para muestras independientes en función de la asistencia a centros bilingüesno bilingües en rendimiento (medido con la prueba de diagnóstico de $4^{\circ}$ de E.P.).

Se observa en la Tabla VII la no existencia de diferencias estadísticamente significativas entre los colegios bilingües y los no bilingües en las pruebas externas realizadas a los alumnos de $4^{\circ}$ de Educación Primaria en Matemáticas y Lengua Castellana debido a que el nivel de significación obtenido es mayor que el alpha preestablecido. 
Resultados basados en las pruebas CDI de $6^{\circ}$ E.P.

\begin{tabular}{lcccc}
\hline & \multicolumn{2}{c}{$\begin{array}{c}\text { Lengua } \\
\text { CDI * }\end{array}$} & \multicolumn{2}{c}{$\begin{array}{c}\text { Matemáticas } \\
\text { CDI * }\end{array}$} \\
\hline Bilingüe & $\mathrm{N}$ & Media & $\mathrm{N}$ & Media \\
No bilingüe & 151 & 14,6 & 146 & 10,3 \\
\hline
\end{tabular}

Nota: $\mathrm{El} *$ indica que la diferencia de medias es significativa al nivel de confianza del $95 \%$. Tabla VIII. Estadísticos de grupo en función de la variable bilingüe-no bilingüe en rendimiento (medido con la prueba CDI).

Prueba para muestras independientes

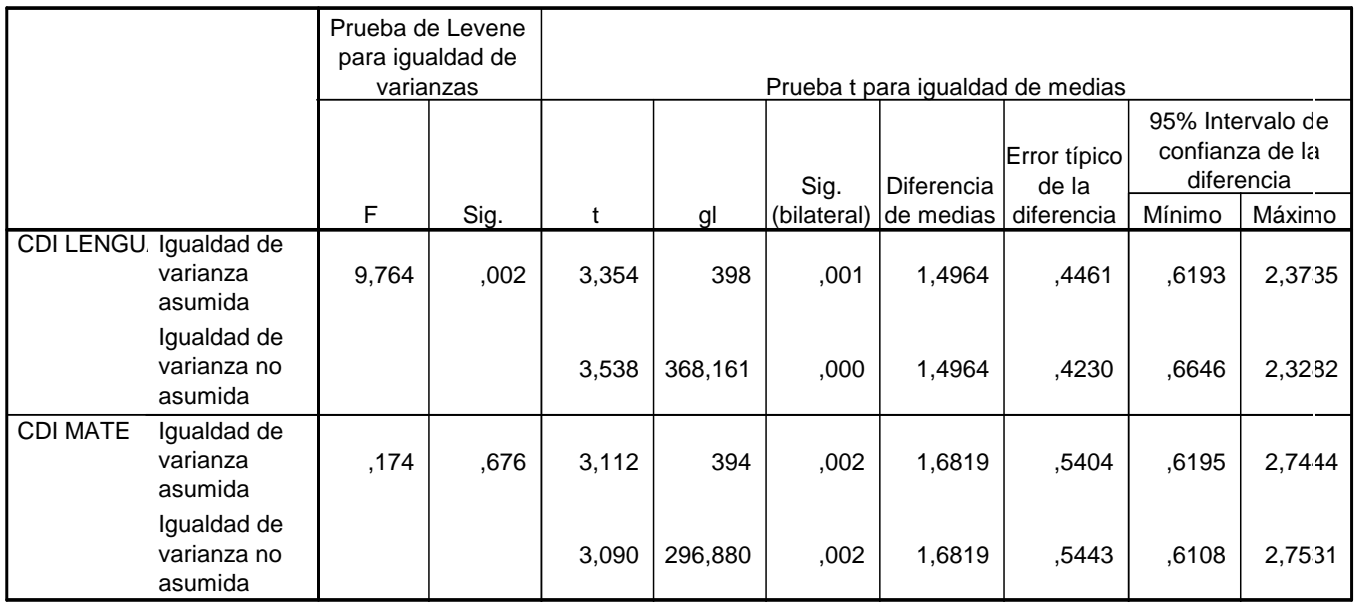

Tabla IX. Prueba para muestras independientes en función de la asistencia a centro bilingüe-no bilingüe en rendimiento (medido con la prueba $\mathrm{CDI}$ ).

Tal y como se observa en la Tabla IX, existen diferencias significativas $(p<, 05)$ entre los colegios bilingües y los no bilingües en las pruebas externas realizadas a los alumnos de $6^{\mathbf{o}}$ de Educación Primaria en Matemáticas (,002) y Lengua Castellana (,001) dado que el nivel de significación obtenido es menor que el alpha preestablecido, observando que los colegios bilingües obtienen unas puntuaciones más altas (en la Tabla VIII los centros bilingües tienen en Matemáticas una media de 10,3 y en Lengua de 14,6).

\section{Conclusiones}

En el presente estudio se ha examinado la repercusión que puede tener la escolarización del alumnado en centros públicos bilingües en el rendimiento académico a través de los resultados escolares obtenidos en las áreas instrumentales (Lengua y 
Matemáticas), en Conocimiento del Medio (debido a su impartición en inglés) y en el área de Inglés, así como a través de la realización de pruebas externas.

Como se ha observado en los resultados de la investigación, si se tienen en cuenta las calificaciones escolares (actas e informes de evaluación final) para evaluar el rendimiento escolar en alumnos del segundo y tercer ciclo de E.P. en función de si asisten a centros públicos bilingües o no bilingües de la Comunidad de Madrid, se hallan diferencias estadísticamente significativas en las áreas de Conocimiento del Medio e Inglés, siendo los colegios no bilingües los que tienen las puntuaciones más altas.

Sin embargo, en referencia a la evaluación del rendimiento escolar mediante las pruebas externas, en $4^{\circ}$ de E.P. no se han hallado diferencias estadísticamente significativas en función de si los alumnos asisten a centros públicos bilingües o no bilingües de la C.M. Por el contrario, en $6^{\circ}$ de E.P. existen diferencias estadísticamente significativas en rendimiento escolar medido con la prueba CDI en Lengua y en Matemáticas a favor de los alumnos de los colegios públicos bilingües. De este modo, evaluando el rendimiento académico mediante las pruebas externas, se puede señalar que en $6^{\circ}$ de E.P., rinden mejor los alumnos de los centros bilingües que los alumnos de los centros no bilingües.

Los resultados hallados en relación con las calificaciones escolares podrían deberse a que en los centros bilingües el profesorado exige un mayor nivel en las áreas de Conocimiento del Medio e Inglés debido a que esas dos áreas se imparten precisamente en inglés, y un centro bilingüe lógicamente debería exigir más. Sin embargo, Lengua y Matemáticas no se ven afectadas negativamente por el hecho de estar escolarizado en un centro bilingüe, de hecho en Matemáticas hay diferencias a favor de los bilingües.

Por otro lado, la obtención de mejores resultados en la prueba externa de $6^{\circ}$ por parte de los centros bilingües, puede mostrar que efectivamente estos alumnos tienen un mayor rendimiento en Lengua y Matemáticas que los alumnos escolarizados en centros monolingües, al menos evaluados mediante pruebas externas. Además, estos resultados se repiten en el estudio realizado por el Consejo Escolar de la C.M. en 2010, en el que se halló que el rendimiento de los Colegios bilingües en la prueba CDI fue significativamente superior, en promedio, a los no bilingües, para los distritos municipales de Madrid.

En referencia a la no obtención de diferencias significativas en la prueba de diagnóstico de $4^{\circ}$ de E.P., quedan respaldados los resultados en el hecho de que las investigaciones coinciden en señalar que "hacia el tercer o cuarto año, los estudiantes del programa de inmersión/bilingües típicamente empiezan a alcanzar, y hacia el quinto año empiezan a superar a todos los grupos de comparación y permanecen obteniendo siempre excelentes resultados académicos" (Thomas, Collier y Abbott, 1993, p. 174). Esta afirmación también coincide con los estudios de Collier (1989), Cummins y Swain (1986) y Genesee (1987), quienes exponen que hacia el cuarto año de inmersión los alumnos de programas bilingües igualan a los de programas monolingües, pero a partir del quinto año comienzan a superar a los monolingües. Sin embargo, muchos estudiantes adquieren competencia como un nativo en destrezas de 
audición y lectura en el idioma meta, pero nunca alcanzan la completa competencia como un nativo en las destrezas productivas de habla y escritura (Collier, 1992).

El presente estudio tiene una justificación empírica y práctica, pero su mayor importancia reside en los aspectos más prácticos de la misma.

No obstante son varias las limitaciones halladas en este trabajo, las investigaciones futuras podrían ir encaminadas a solventar las mismas.

La primera limitación está relacionada con el tiempo, debido a que se debería realizar un estudio en un largo período de tiempo (una investigación longitudinal en la que se recogieran los resultados de las pruebas externas y las calificaciones escolares todos los años) y en una amplia variedad de contextos (ampliar a las distintas Direcciones de Área Territoriales y a Educación Secundaria).

Otra limitación es el hecho de no incluir el nivel de dominio de la lengua inglesa (evaluado éste a través de una prueba externa). Como propuesta de futuro habría que evaluar el rendimiento en Inglés y en Conocimiento del Medio con pruebas estandarizadas.

A pesar de todo, se considera que es un primer acercamiento para determinar las relaciones entre el bilingüismo español-inglés y su relación con el rendimiento académico, pero única y exclusivamente en relación a los alumnos que han formado parte del estudio. No se han intentado generalizar, en ningún momento, los resultados obtenidos como características propias de la población a la que pertenecen dichos sujetos.

\section{Referencias bibliográficas}

BARIK, H. C. \& SWAIN, M. (1978). Evaluation of a French immersion program: The Ottawa study through grade five. Canadian Journal of Behavioural Science, 10 (3), 192-201.

BIALYSTOK, E. (1986). Factors in the growth of linguistic awareness. Child Development, 57, 498-510.

BIALYSTOK, E. (1997). Effects of bilingualism and biliteracy on children's emerging concepts of print. Developmental Psychology, 33 (3), 429-440.

BIALYSTOK, E. \& MAJUMDER, S. (1998). The relationship between bilingualism and the development of cognitive processes in problem solving. Applied Psycholinguistics, 19, 69-85.

BOURNOT-TRITES, M. \& REEDER, K. (2001). Interdependence Revisited: Mathematics Achievement in an Intensified French Immersion Program. Canadian Modern Language Review, 58 (1), 27-43.

CADE, J. M. (1997). The foreign language immersion program in the Kansas City, Missouri Public Schools, 1986-1996 [Abstract]. Dissertation Abstracts International -A 58(10), 3838. 
COHEN, A. D. (1974). The Culver CitySpanish immersion program: The first two years. The Modern Language Journal, 58 (3), 95-103.

COLLIER, C. (1989). How Long? A Synthesis of Research on Academic Achievement in a Second Language. TESOL Quarterly, 23 (3), 509-531.

COLLIER, V. P. (1992). The Canadian Bilingual Immersion Debate: A Synthesis of Research Findings. Studies in Second Language Acquisition 14, 87-97.

CONSEJO ESCOLAR. COMUNIDAD DE MADRID. (2010). Los Programas de Enseñanza Bilingüe de la Comunidad de Madrid. Un estudio comparado. Recuperado de http:/www.madrid.org/edupubli (Biblioteca Virtual Consejería de Educación de la C.M.)

CROMDAL, J. (1999). Childhood bilingualism and metalinguistic skills: Analysis and control in young Swedish-English bilinguals. Applied Psycholinguistics, 20, 1-20.

CUMMINS, J. (2001). ¿Qué sabemos de la educación bilingüe?: Perspectivas psicolingüísticas y sociológicas. Revista de educación, 326, 37-61.

CUMMINS, J. (2002). Lenguaje, poder y pedagogía: Niños y niñas bilingües entre dos fuegos. MEC: Morata.

CUMMINS, J. (2010). Teaching for Transfer in Bilingual Education: Promoting Language Awareness and Literacy Engagement through Identity Texts. I Congreso Internacional sobre Bilingüismo en Centros Educativos. Madrid.

CUMMINS, J. \& SWAIN, M. (1986). Bilingualism in Education. New York: Longman.

D'ANGIULLI, A., SIEGEL, L. S. \& SERRA, E. (2001). The development of reading in English and Italian in bilingual children. Applied Psycholinguistics, 22 (4), 479507.

DEMONT, E. (2001). Contribution of early 2nd-language learning to the development of linguistic awareness and learning to read/Contribution de l'apprentissage précoce d'une deuxième langue au développment de la conscience lingustique et à l'apprentissage de la lecture. International Journal of Psychology, 36 (4), 274-285.

EDWARDS, H. (1989). Review of the literature. In N. Halsall, Immersion/regular program study (pp. 1-64). Nepean, ON: Carleton Board of Education.

FEDERACIÓN DE LA COMUNIDAD DE MADRID DE APAS FRANCISCO GINER DE LOS RÍOS. (2009). Informe sobre el Programa de Centros bilingües de la Comunidad de Madrid.

GALINDO, A. \& MORENO, L. M. (2008). L1 Argumentative Written Production in Bilingual and Monolingual Contexts in 6th, 7th and 8th Grades. Íkala, 13 (19), 145169.

GENESEE, F. (1987). Learning through Two Languages: Studies of Immersion and Bilingual Education. Cambridge, MA: Newbury House. 
GENESEE, F. \& LAMBERT, W. E. (1983). Trilingual education for majoritylanguage children. Child Development, 54 (1), 105-114.

GENESEE, F., LAMBERT, W. E. \& HOLOBOW, N. E. (1986). La adquisición de una segunda lengua mediante inmersión: el enfoque canadiense. Infancia y aprendizaje, $33,27-36$.

HALSALL, N. (1998). French immersion: The success story told by research. Keynote address at "French Immersion in Alberta: Building the Future Conference", Edmonton, Alberta, November 19-20, 1998.

HAWSON, A. (1996). A neuroscientific perspective on second-language learning and academic achievement. Bilingual Review, 21 (2), 101-123.

HOLOBOW, N. E., GENESEE, F., LAMBERT, W. E. \& GASTRIGHT, J. (1987). Effectiveness of partial French immersion for children from different social class and ethnic backgrounds. Applied Psycholinguistics, 8 (2), 137-151.

JOHNSTONE, R., DOBSON, A. \& PÉREZ MURILLO, M. D. (2010). BEP (Spain) EVALUATION. Documento elaborado por el equipo de evaluación para la presentación celebrada el 25 de marzo de 2010. Madrid.

KESSLER, C. \& QUINN, M. E. (1980). Positive effects of bilingualism on Science problem-solving abilities. In J. Alatis (Ed.), Georgetown University round table on languages and linguistics (pp. 295-308). Washington, DC: Georgetown University Press.

KIMBROUGH OLLER, D. \& COBO-LEWIS, A. (2002). The Ability of Bilingual and Monolingual children to Perform Phonological Translation. In D. Kimbrough Oller and R.E. Eilers (Eds.), Language and Literacy in Bilingual Children (pp. 255-277). Clevedon, England: Multilingual Matters.

LAMBERT, W. E., GENESEE, F., HOLOBOW, N. \& CHARTRAND, L. (1993). Bilingual education for majority English-speaking children. European Journal of Psychology of Education, 8 (1), 3-22.

LAPKIN, S. (1982). The English writing skills of French immersion pupils at grade five. La Revue Canadienne des Langues Vivantes, 39, 24-33.

LAPKIN, S., SWAIN, M. \& SHAPSON, S. (1990). French immersion research agenda for the 90s. The Canadian Modern Language Review, 46 (4), 638-674.

LASAGABASTER, D. (2005). La presencia de tres lenguas en el currículo: Multilingüismo en los contextos canadiense y español. Revista de educación, 337, 405-426.

ORDEN 796/2004 de 5 de marzo, de la Consejería de Educación de la Comunidad de Madrid, para la selección de colegios públicos de Educación Infantil y Primaria de la Comunidad de Madrid en los que se llevará a cabo la implantación de la enseñanza bilingüe español-inglés. 
PAGAN, C. R. (2005). English learners' academic achievement in a two-way versus a structured English immersion program. Unpublished doctoral dissertation, Teachers College, New York.

PIFARRÉ TURMO, M., SANUY BURGUÉS, J., HUGUET CANALIS, A. \& VENDRELL SERÈS, C. (2003).Rendimiento matemático en contextos bilingües: Análisis de la incidencia de algunas variables del contexto socio-educativo. Revista de Investigación Educativa, 21 (1), 183-199.

RESOLUCIÓN de 15 de marzo de 2010, de las Viceconsejerías de Educación y de Organización Educativa, por la que se dictan instrucciones para la celebración de la prueba de conocimientos y destrezas indispensables de los alumnos de sexto curso de Educación Primaria de la Comunidad de Madrid, en el curso 2009-2010.

RESOLUCIÓN de 26 de marzo de 2010, de la Dirección General de Mejora de la Calidad de la Enseñanza, por la que se dictan instrucciones para la evaluación de diagnóstico de cuarto curso de Educación Primaria, que se realizará en el curso 2009-2010.

RICCIARDELLI, L. A. (1993). An investigation of the cognitive development of Italian-English bilinguals and Italian monolinguals from Rome. Journal of Multilingual and Multicultural Development, 14 (4), 345-346.

SWAIN, M. (1987). French immersion: Early, late or partial? La Revue Canadienne des Langues Vivantes, 34 (3), 577-585.

SWAIN, M. \& LAPKIN, S. (1981). Bilingual education in Ontario: A decade of research. Toronto: Ontario Institute for Studies in Education.

SWAIN, M. \& LAPKIN, S. (1982). Evaluating Bilingual Education: A Canadian Case Study. Clevedon, England: Multilingual Matters.

THOMAS, W. P. \& COLLIER, V. P. (2003). The Multiple Benefits of Dual Language. Educational Leadership, 61 (2), 61-64.

THOMAS, W. P., COLLIER, V. P. \& ABBOTT, M. (1993). Academic achievement through Japanese, Spanish, or French: The first two years of partial immersion. Modern Language Journal, 77 (2), 170-179.

TURNBULL, M., HART, D. \& LAPKIN, S. (2003). Grade 6 French immersion students' performance on large-scale reading, writing, and mathematics tests: Building explanations. Alberta Journal of Educational Research, 49 (1), 6-23.

TURNBULL, M., LAPKIN, S. \& HART, D. (2001). Grade 3 immersion students' performance in literacy and mathematics: Province-wide results from Ontario (1998-99). La Revue Canadienne des Langues Vivantes, 58 (1), 9-26.

WAYNE, T. \& COLLIER, V. (2002). A National Study of School Effectiveness for Language Minority Students' Long-Term Academic Achievement. Center for Research on Education, Diversity and Excellence. UC Berkeley. 


\section{AGRADECIMIENTOS}

Quiero expresar mi más sincera gratitud a los centros participantes en el estudio, en especial a los equipos directivos. A Coral y a Ana les agradezco sus valiosas sugerencias y aportaciones, muchísimas gracias por vuestra ayuda incondicional. A vosotras y a mi familia os dedico este trabajo.

Parte de esta investigación se llevó a cabo durante el disfrute de una licencia por estudios concedida por la Consejería de Educación durante el curso 2009/10.

\section{Correspondencia con la autora}

\section{Esmeralda SOTOCA SIENES}

CEIP Ángel Berzal Fernández

Camino de Fresno, s/n (28814)

Teléfono: 918841258

e-mail: esmeralda.sotocasienes@educa.madrid.org 